SNR-Aided Accurate

\section{Phase Estimation in 1-Bit Software-Defined Receiver}

\author{
WAN-HSIN HSIEH \\ National Chiao Tung University \\ CHIEH-FU CHANG \\ National Space Organization
}

MING-SENG KAO, Member, IEEE

National Chiao Tung University

This work investigates the high-accuracy phase discriminator in 1-bit software-defined receiver (SDR) for three signal-to-noise ratio (SNR) ranges: low SNR, high SNR and moderate SNR. Unlike traditional approaches, our approach first distinguishes which SNR range an application falls into, and this SNR information is then utilized to select a proper phase discriminator to achieve high accuracy. For low-SNR applications, traditional arctangent phase discriminator (APD) is adopted and the analysis of asymptotic performance is derived. For high-SNR applications, the noise-balanced digital phase discriminator (NB-DPD) is adopted to improve accuracy [1]. Between them, for moderate-SNR applications, a novel SNR-aided phase discriminator (SNRaPD) is proposed. The analytical and simulation results verify the superiority of the proposed approach for applications in different SNR ranges. The determination of moderate SNR range is critical and discussed finally.

Manuscript received February 25, 2010; revised December 7, 2010 and May 13, 2011; released for publication May 24, 2011.

IEEE Log No. T-AES/48/2/943835.

Refereeing of this contribution was handled by J. Morton.

Authors' addresses: W-H. Hsieh and M-S. Kao, Department of Electrical Engineering, National Chiao Tung University, Lab. 717, Engineering Building 4, No. 1001, Daxue Rd., Hsinchu, 30010, Taiwan, E-mail: (hsieh1979@gmail.com); C-F. Chang, Electrical Engineering Division, National Space Organization, Hsinchu, Taiwan.

$\overline{0018-9251 / 12 / \$ 26.00 ~(C) ~} 2012$ IEEE

\section{INTRODUCTION}

In discrete phase estimation, the arctangent phase discriminator (APD) is widely adopted since it achieves maximum likelihood estimation (MLE) in additive white Gaussian noise (AWGN) [2, p. 167]. With full precision, i.e., infinite analog-to-digital conversion (ADC) bits, the APD attains MLE no matter what the signal-to-noise ratio (SNR) is. However, this is not the case for realistic phase discriminators that have finite precision using few-bit ADC, especially those with 1-bit ADC. In this work, we study the accurate 1-bit carrier phase discriminator and propose an SNR-aided approach to enhance the ultimate accuracy in 1-bit software-defined receiver (SDR). Note that both the received signal and local reference signal are 1-bit quantized before the digital computations in SDR. Analyses of asymptotic performance and the relationship between the phase estimation accuracy and the SNR are also provided.

In this 1-bit scenario, efficient bitwise processing, e.g., multiplication becomes exclusive-OR operation, can be adopted to greatly reduce computational load, and the required storage of local reference signal is also minimized. Because of efficient bitwise processing and the avoidance of automatic gain control (AGC) in modern applications [3-7], the estimation using 1-bit ADC has induced wide interest. The prior researches related to our work are introduced as follows. First, the problem of parameter estimation for a single sinusoid was previously investigated in [8]-[11]. In [11], Cramér-Rao bound (CRB) of 1-bit quantization could be derived under the assumption of independence between quantized samples. The effects of 1-bit sampling and quantization were also discussed. Unfortunately, due to the lack of a closed form of probability mass function of samples [11, eq. (10)], the derivation of MLE of carrier is intractable. Next, the dithering techniques were used to improve the estimation performance. In [12] and [13], the asymptotic bias of 1-bit quantized mean estimation problems was addressed. Other relevant studies fell in the field of the limiter phase detector $[14,15$, ch. 10], which utilizes a limiter to prevent overload of the received signal. As we consider a limiter as a binary quantizer with infinite samples, some results in this field can be used to verify the asymptotic results of our work in low SNR. For high SNR, the asymptotic phase estimation bias of APD has been mentioned and an improved phase discriminator, called digital phase discriminator (DPD), is proposed in [16]. The proposed DPD achieves much higher asymptotic accuracy than that of the traditional APD. However, the DPD does not perform well 


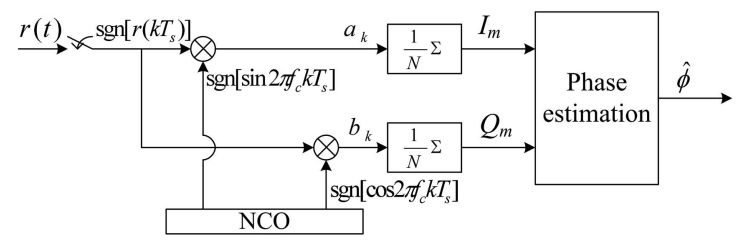

Fig. 1. System structure of 1-bit SDR.

in low SNR environments due to its sensitivity to noise. For spaceborne measurement techniques utilizing accurate phase estimation in SDR, e.g. total electron content (TEC) measurements and the precise orbit determination (POD) [19-23], where the ambient SNR may vary widely, performance can be further improved by including the SNR in the phase estimation.

In this paper, the traditional inphase-quadrature (I-Q) structure using 1-bit ADC is investigated and the SNR-dependent mean value of the I-Q channel output is studied in Section II. In Section III, the phase estimation with APD and a modified DPD (the noise-balanced digital phase discriminator (NB-DPD)) are described. The SNR-aided phase discriminator (SNRaPD) scheme is then presented, and the improvement in accuracy is illustrated in Section IV. The performance of SNRaPD is simulated and compared with the average CRB. In addition, an adequate stop criterion and the range of applications regarding SNR of SNRaPD are also discussed. It is followed by the conclusions in Section V.

\section{SYSTEM MODEL}

The system model for carrier phase estimation in the 1-bit SDR is shown Fig. 1. The received signal is denoted by

$$
r(t)=\sin \left(2 \pi f_{c} t+\phi\right)+\nu(t)
$$

where $f_{c}$ is the carrier frequency, $\phi$ is an unknown phase, and $\nu(t)$ is the AWGN. Since the phase estimation is of interest, the output frequency of the numerically controlled oscillator (NCO) is assumed equal to the incoming carrier frequency. Let $\mathrm{T}_{\mathrm{s}}$ be the sampling period. The discrete-time 1-bit quantized $r(t)$ is given by

$$
\begin{aligned}
r[k] & =\operatorname{sgn}\left[r\left(k \mathrm{~T}_{\mathrm{s}}\right)\right] \\
& =\operatorname{sgn}\left[\sin \left(2 \pi f_{c} k \mathrm{~T}_{\mathrm{s}}+\phi\right)+\nu\left(k \mathrm{~T}_{\mathrm{s}}\right)\right]
\end{aligned}
$$

where

$$
\operatorname{sgn}[x]=\left\{\begin{array}{lll}
1 & \text { if } \quad x \geq 0 \\
-1 & \text { if } \quad x<0
\end{array} .\right.
$$

In addition, let the sampling frequency be $f_{s}=1 / \mathrm{T}_{\mathrm{s}}$. We denote

$$
\frac{f_{c}}{f_{s}}=h+\frac{q}{p}
$$

where $h$ is the greatest integer less than or equal to $f_{c} / f_{s}$, and $p$ and $q$ are mutually prime integers. The mixer output of the inphase channel is given by

$$
\begin{aligned}
a_{k} & =\operatorname{sgn}\left[r\left(k \mathrm{~T}_{\mathrm{s}}\right)\right] \cdot \operatorname{sgn}\left[\sin 2 \pi f_{c} k \mathrm{~T}_{\mathrm{s}}\right] \\
& =\operatorname{sgn}\left[\sin \left(\Phi_{k}+\phi\right)+\nu_{k}\right] \cdot \operatorname{sgn}\left[\sin \Phi_{k}\right]
\end{aligned}
$$

where $\Phi_{k}=2 \pi f_{c} k \mathrm{~T}_{\mathrm{s}}$, and $\nu_{k}=\nu\left(k \mathrm{~T}_{\mathrm{s}}\right)$ is a zero-mean Gaussian random variable with variance $\sigma^{2}$.

Consider the $p$ samples at $t=k \mathrm{~T}_{\mathrm{s}}, k=$ $0,1,2, \ldots, p-1$. The normalized I-Q channel outputs are, respectively, denoted by

$$
\begin{aligned}
I_{p} & =\frac{1}{p} \sum_{k=0}^{p-1} \operatorname{sgn}\left[\sin \left(\Phi_{k}+\phi\right)+\nu_{k}\right] \cdot \operatorname{sgn}\left[\sin \Phi_{k}\right] \\
Q_{p} & =\frac{1}{p} \sum_{k=0}^{p-1} \operatorname{sgn}\left[\sin \left(\Phi_{k}+\phi\right)+\nu_{k}\right] \cdot \operatorname{sgn}\left[\cos \Phi_{k}\right] .
\end{aligned}
$$

It can be proved that the samples of phases $\left\{\Phi_{0}, \Phi_{1}, \ldots, \Phi_{p-1}\right\}$ are uniformly distributed over $[0,2 \pi)$ with a separation of $2 \pi / p$ between neighboring $\Phi_{i} \mathrm{~s}$ [16]. Note that when we mention that $p$ is "sufficiently" large later, it means that $2 \pi / p$ is significantly smaller than the accuracy required in the estimation. According to Appendix I, the mean values and variances of the I-Q channel outputs are given by

$$
\begin{aligned}
& \mu_{I_{p}}=\frac{1}{p}\left[\sum_{\Phi_{k} \in[0, \pi)}\left(1-2 \mathrm{P}_{k}\right)+\sum_{\Phi_{k} \in[\pi, 2 \pi)}\left(2 \mathrm{P}_{k}-1\right)\right] \\
& \mu_{Q_{p}}=\frac{1}{p}\left[\sum_{\Phi_{k} \in[0, \pi / 2) \cup[3 \pi / 2,2 \pi)}\left(1-2 \mathrm{P}_{k}\right)+\sum_{\Phi_{k} \in[\pi / 2,3 \pi / 2)}\left(2 \mathrm{P}_{k}-1\right)\right] \\
& \sigma_{I_{p}}^{2}=\sigma_{Q_{p}}^{2}=\frac{4}{p^{2}} \sum_{k=0}^{p-1} \mathrm{P}_{k}-\mathrm{P}_{k}^{2} .
\end{aligned}
$$

where

$$
\begin{aligned}
\mathrm{P}_{k} & =\mathrm{Q}\left(\frac{\sin \left(\Phi_{k}+\phi\right)}{\sigma}\right) \\
\mathrm{Q}(x) & =\frac{1}{\sqrt{2 \pi}} \int_{x}^{\infty} \exp \left(-\frac{z^{2}}{2}\right) d z .
\end{aligned}
$$

Note that the range of summation is defined according to the value of $\Phi_{k}$ in (6), and $\mathrm{P}_{k}$ is a function of $\Phi_{k}$. In (7), the variance of the 1-bit quantized I-Q outputs consists of the effect of channel noise and quantization noise. Since $\left\{\Phi_{0}, \Phi_{1}, \ldots, \Phi_{p-1}\right\}$ are uniformly distributed over $[0,2 \pi)$, the mean value of I-channel output in (6a) can be further derived by 


$$
\begin{aligned}
\mu_{I_{p}} & =\frac{1}{p}\left[\frac{p}{2}-\sum_{\Phi_{k} \in[0, \pi)} 2 \mathrm{Q}\left(\frac{\sin \left(\Phi_{k}+\phi\right)}{\sigma}\right)+\sum_{\Phi_{k} \in[\pi, 2 \pi)} 2 \mathrm{Q}\left(\frac{\sin \left(\Phi_{k}+\phi\right)}{\sigma}\right)-\frac{p}{2}\right] \\
& =\frac{1}{p}\left[\sum_{\Phi_{k} \in[\pi, 2 \pi)} 2 \mathrm{Q}\left(\frac{\sin \left(\Phi_{k}+\phi\right)}{\sigma}\right)-\sum_{\Phi_{k} \in[0, \pi)} 2 \mathrm{Q}\left(\frac{\sin \left(\Phi_{k}+\phi\right)}{\sigma}\right)\right] .
\end{aligned}
$$

From (1), the SNR of the sinusoidal signal is given by

$$
\mathrm{SNR}=\frac{1}{2 \sigma^{2}} .
$$

Equation (8) can then be written as

$\mu_{I_{p}}=\frac{1}{p}\left[\sum_{\Phi_{k} \in[\pi, 2 \pi)} 2 \mathrm{Q}\left(\gamma \sin \left(\Phi_{k}+\phi\right)\right)-\sum_{\Phi_{k} \in[0, \pi)} 2 \mathrm{Q}\left(\gamma \sin \left(\Phi_{k}+\phi\right)\right)\right]$

where $\gamma=\sqrt{2 \mathrm{SNR}}$.

Suppose we choose $f_{s}$ such that $p$ is sufficiently large in (3). According to Appendix II, the mean value of I-channel output of (10) can be represented as a power series, which is given by

$$
\begin{aligned}
\mu_{I_{p}}= & A \sum_{m=0}^{\infty} \frac{\gamma^{2 m+1}}{m ! 2^{3 m}(2 m+1)} \\
& \times\left[\sum_{l=0}^{m}(-1)^{l}\left(\begin{array}{c}
2 m+1 \\
l
\end{array}\right) \frac{\cos (2 m+1-2 l) \phi}{2 m+1-2 l}\right]
\end{aligned}
$$

where $A=4 / \sqrt{2} \pi^{3 / 2}$.

Similarly, the mean value of Q-channel output of $(6 b)$ is denoted by

$$
\begin{aligned}
\mu_{Q_{p}}=\frac{1}{p} & {\left[\sum_{\Phi_{k} \in[0, \pi / 2) \cup[3 \pi / 2,2 \pi)}-2 \mathrm{Q}\left(\gamma \sin \left(\Phi_{k}+\phi\right)\right)\right.} \\
& \left.+\sum_{\Phi_{k} \in[\pi / 2,3 \pi / 2)} 2 \mathrm{Q}\left(\gamma \sin \left(\Phi_{k}+\phi\right)\right)\right] .
\end{aligned}
$$

By a similar derivation of (11), the power series representation of $\mu_{Q}$ is given by

$$
\begin{aligned}
\mu_{Q_{p}}= & A \sum_{m=0}^{\infty} \frac{(-1)^{m} \gamma^{2 m+1}}{m ! 2^{3 m}(2 m+1)} \\
& \times\left[\sum_{l=0}^{m}\left(\begin{array}{c}
2 m+1 \\
l
\end{array}\right) \frac{\sin (2 m+1-2 l) \phi}{2 m+1-2 l}\right] .
\end{aligned}
$$

In addition, according to Appendix II, the power series of the variances of the I-Q channel outputs of
(7) are represented by

$$
\begin{aligned}
& \sigma_{I_{p}}^{2}=\sigma_{Q_{p}}^{2} \\
&=\frac{1}{p}-\frac{2}{p \pi}\left[\sum_{m=0}^{\infty}\left(\begin{array}{c}
4 m+2 \\
2 m+1
\end{array}\right) \frac{\gamma^{4 m+2}}{\left(m ! 2^{3 m+1}(2 m+1)\right)^{2}}\right. \\
&+2 \sum_{x=0}^{\infty} \sum_{y=x+1}^{\infty}\left(\begin{array}{c}
2 x+2 y+2 \\
x+y+1
\end{array}\right) \\
&\left.\quad \times \frac{(-1)^{x+y} \gamma^{2(x+y)+2}}{x ! y ! 2^{3(x+y)+2}(2 x+1)(2 y+1)}\right] .
\end{aligned}
$$

The above results are obtained for $p$ samples. When the mean values and variances are generalized to $N=$ $m p$ samples, where $m$ is an integer, the normalized I-Q channel outputs are given by

$$
\begin{aligned}
I_{m} & =\frac{1}{N} \sum_{k} \operatorname{sgn}\left[\sin \left(\Phi_{k}+\phi\right)+\nu_{k}\right] \cdot \operatorname{sgn}\left[\sin \Phi_{k}\right] \\
Q_{m} & =\frac{1}{N} \sum_{k} \operatorname{sgn}\left[\sin \left(\Phi_{k}+\phi\right)+\nu_{k}\right] \cdot \operatorname{sgn}\left[\cos \Phi_{k}\right] .
\end{aligned}
$$

The mean values of the I-Q channel outputs are the same as (11) and (13), respectively. The variance can be expressed as

$$
\begin{aligned}
\sigma_{I}^{2}=\sigma_{Q}^{2} & \\
=\frac{1}{N}-\frac{2}{N \pi} & {\left[\sum_{m=0}^{\infty}\left(\begin{array}{c}
4 m+2 \\
2 m+1
\end{array}\right) \frac{\gamma^{4 m+2}}{\left(m ! 2^{3 m+1}(2 m+1)\right)^{2}}\right.} \\
& +2 \sum_{x=0}^{\infty} \sum_{y=x+1}^{\infty}\left(\begin{array}{c}
2 x+2 y+2 \\
x+y+1
\end{array}\right) \\
& \left.\times \frac{(-1)^{x+y} \gamma^{2(x+y)+2}}{x ! y ! 2^{3(x+y)+2}(2 x+1)(2 y+1)}\right] .
\end{aligned}
$$

The relationship between the mean values of the I-Q channel outputs is shown in Fig. 2. Here, only the relationship in the 1 st quadrant is illustrated because of the symmetry of the trigonometric function. As can be seen in Fig. 2, the relationship between the I-Q channel outputs varies with SNR. The relationship 


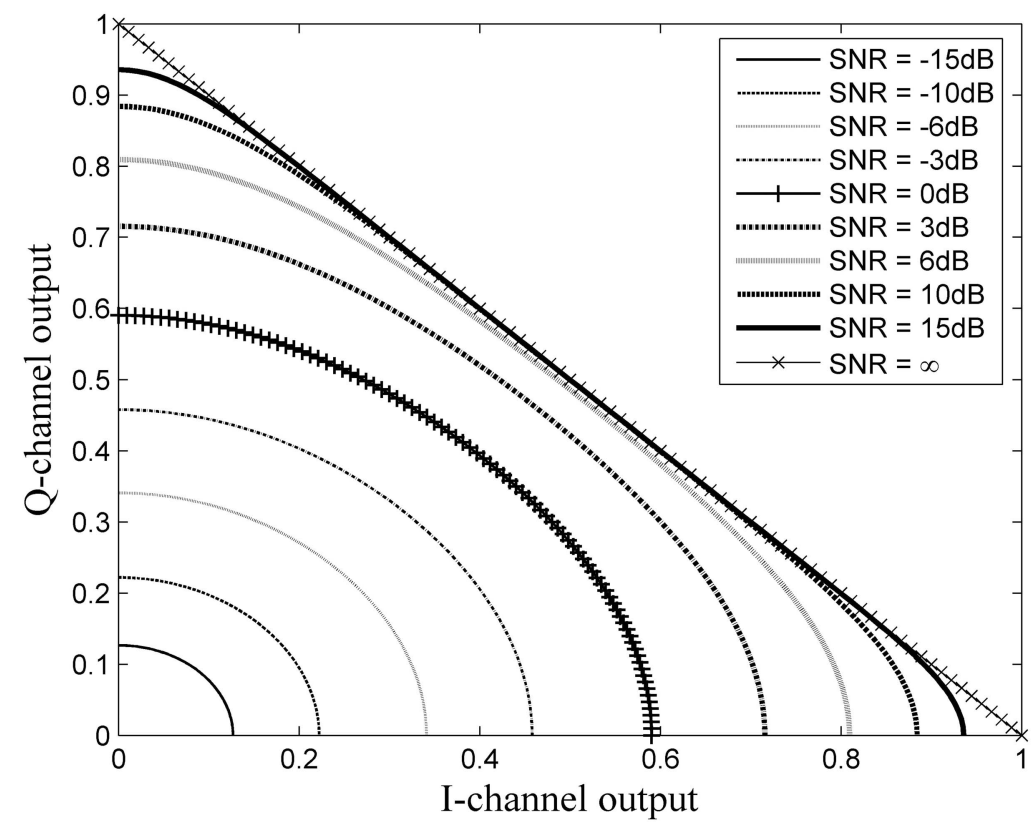

Fig. 2. Relationship between mean values of I-Q channel outputs (first quadrant).

approximates a circle when the SNR is low, but deviates from a circle with increasing SNR. When $\mathrm{SNR} \rightarrow \infty$, the relationship becomes a straight line. Since the phase is estimated from the I-Q channel outputs in Fig. 1, the phase estimation should be adjusted according to the SNR to achieve high accuracy. In the following sections, accurate phase estimations regarding SNR are introduced. Note that in order to evaluate the achievable phase accuracy, the assumption of zero frequency offset is inherently applied to the following analyses and simulations.

\section{ACCURATE 1-BIT PHASE DISCRIMINATOR}

In this section, we introduce phase discriminators that have been proposed in the literature, i.e., DPD [16], NB-DPD [1], and APD, for 1-bit quantized data and discuss their performance regarding SNR.

\section{A. High SNR}

When SNR $\rightarrow \infty, \nu_{k}$ in (15) can be neglected. Moreover, when the number of samples $N$ is large, by law of large numbers (LLN), the I-Q channel outputs, denoted as $\left(I_{m}, Q_{m}\right)$, approximate their mean values, i.e., $I_{m} \rightarrow \mu_{I}$ and $Q_{m} \rightarrow \mu_{Q}$. The relationship between the I-Q channel outputs is then denoted by

$$
\mu_{I}+\mu_{Q}=1 .
$$

The relationship is a straight line as shown in Fig. 2. In this situation, the phase can be estimated by DPD to achieve higher accuracy than that of APD [16]. Applying the system model of Fig. 1 in [16], the phase estimation according to $\left(I_{m}, Q_{m}\right)$ is given by

$$
\hat{\phi}_{\mathrm{DPD}}=\operatorname{sgn}\left(Q_{m}\right) \frac{\pi}{2}\left(1-I_{m}\right) .
$$

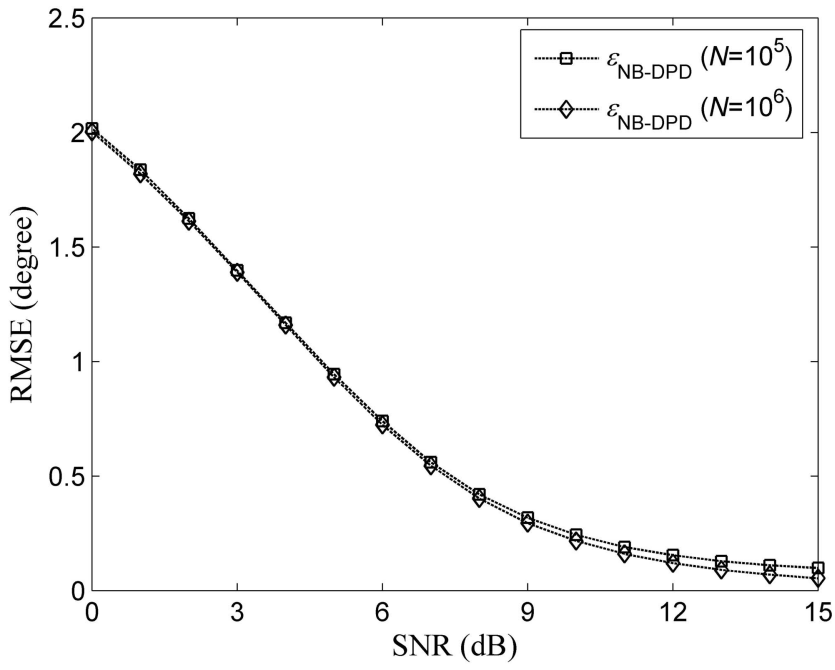

Fig. 3. Simulated results of NB-DPD regarding SNR.

The mean-squared error (MSE) of DPD will be negligible with a sufficiently large $N$.

However, in the high SNR environment, there still exists small noise variance in the I-Q channel outputs, and the relationship between the mean I-Q outputs deviates from a straight line as shown in Fig. 2. Since DPD is sensitive to noise, the modified DPD, called NB-DPD, is provided to improve phase estimation in this situation. The phase estimated by NB-DPD is given by [1]

$$
\hat{\phi}_{\mathrm{NB}-\mathrm{DPD}}=\operatorname{sgn}\left(Q_{m}\right) \frac{\pi}{2}\left(1-\frac{I_{m}}{\left|I_{m}\right|+\left|Q_{m}\right|}\right) .
$$

Let the root mean-squared error (RMSE) of NB-DPD be denoted by $\varepsilon_{\mathrm{NB}-\mathrm{DPD}}=\sqrt{\mathrm{E}\left[\left(\hat{\phi}_{\mathrm{NB}-\mathrm{DBD}}-\phi\right)^{2}\right]}$. Using Monte Carlo simulation methods for 100 trials, 


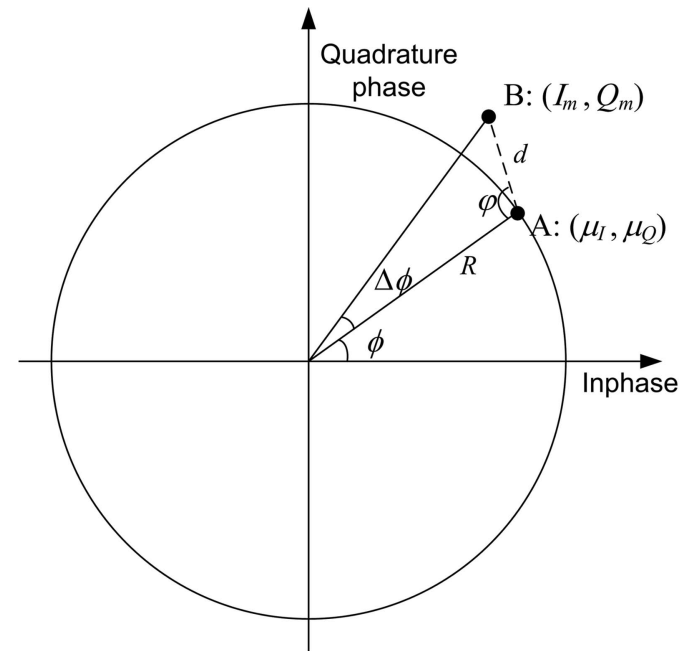

Fig. 4. Geometric representation of performance of APD on I-Q plane.

$\varepsilon_{\text {NB-DPD }}$ regarding SNR is shown in Fig. 3. As expected, $\varepsilon_{\text {NB-DPD }}$ decreases with increasing SNR. In addition, it decreases slightly with $N$ in high SNR. Apparently, the NB-DPD can achieve high phase estimation accuracy in high SNR.

\section{B. Low SNR}

When the SNR is low, we have $\gamma \ll 1$ in (11) and (13). Thus, the mean values of the I-Q channel outputs are approximated by

$$
\begin{aligned}
& \mu_{I}=\frac{4 \gamma}{\sqrt{2} \pi^{3 / 2}} \cos \phi \\
& \mu_{Q}=\frac{4 \gamma}{\sqrt{2} \pi^{3 / 2}} \sin \phi
\end{aligned}
$$

This proves the circular relationship of the I-Q channel output in the low SNR environment as shown in Fig. 2. The results are also consistent with Pouzet's conclusion, when we consider a limiter as a binary quantizer with an infinite number of samples [14, 15, ch. 10]. Since the mean I-Q channel outputs have an approximately circular relationship, APD is a good choice in low SNR. In addition, the variances of the I-Q channel outputs of (16) are approximated by

$$
\begin{aligned}
\sigma_{I}^{2} & =\sigma_{Q}^{2} \\
& =\frac{1}{N}\left(1-\frac{\gamma^{2}}{\pi}\right) \\
& \approx \frac{1}{N}
\end{aligned}
$$

when the SNR is low.

The performance of APD in low SNR can be analyzed using geometry as shown in Fig. 4. First, let point $\mathrm{A}:\left(\mu_{I}, \mu_{Q}\right)$ denote the mean values of the I-Q outputs on a circle with radius $R$ and polar phase $\phi$. According to (20), the radius $R$ is given by

$$
\begin{aligned}
R & =\sqrt{\mu_{I}^{2}+\mu_{Q}^{2}} \\
& =\frac{4 \gamma}{\sqrt{2} \pi^{3 / 2}} \\
& =\frac{4 \sqrt{\mathrm{SNR}}}{\pi^{3 / 2}} .
\end{aligned}
$$

Next, let B : $\left(I_{m}, Q_{m}\right)$ be the measured I-Q channel outputs. The distance between $\mathrm{A}$ and $\mathrm{B}$ is denoted by

$$
d=\sqrt{\left(I_{m}-\mu_{I}\right)^{2}+\left(Q_{m}-\mu_{Q}\right)^{2}} .
$$

The carrier phase estimated from $\left(I_{m}, Q_{m}\right)$ with APD is given by

$$
\hat{\phi}_{\mathrm{APD}}=\operatorname{atan} 2\left(\frac{Q_{m}}{I_{m}}\right)=\phi+\Delta \phi
$$

where $\operatorname{atan} 2(x)$ denotes the arctangent-2 function and $\Delta \phi$ is the phase estimation error. Without loss of generality, let $\Delta \phi \geq 0$. From the law of sines, we have

$$
\frac{d}{\sin \Delta \phi}=\frac{R}{\sin (\pi-\varphi-\Delta \phi)} \text {. }
$$

Assume $\Delta \phi \ll 1$. Then $\sin \Delta \phi \approx \Delta \phi$, and $\sin (\pi-\varphi-\Delta \phi) \approx \sin (\pi-\varphi)$. Equation (25) is approximated by

$$
\Delta \phi \approx \frac{d}{R} \sin (\pi-\phi)
$$

Suppose $\varphi$ is uniformly distributed over $[0, \pi)$. For a fixed $d$, the expected value of $\Delta \phi^{2}$ with respect to $\varphi$ is denoted by

$$
\begin{aligned}
\mathrm{E}_{\varphi}\left[\Delta \phi^{2}\right] & =\frac{1}{\pi} \int_{0}^{\pi} \frac{d^{2}}{R^{2}} \sin ^{2}(\pi-\varphi) d \varphi \\
& =\frac{d^{2}}{2 R^{2}} .
\end{aligned}
$$

Assume $d$ and $\varphi$ are mutually independent. According to the definition of variance and (21), the expected value of $d^{2}$ is given by

$$
\begin{aligned}
\mathrm{E}_{d}\left[d^{2}\right] & =\mathrm{E}\left[\left(I_{m}-\mu_{I}\right)^{2}+\left(Q_{m}-\mu_{Q}\right)^{2}\right] \\
& =\sigma_{I}^{2}+\sigma_{Q}^{2} \\
& =\frac{2}{N} .
\end{aligned}
$$

From (27) and (28), the MSE of phase estimation is denoted by

$$
\begin{aligned}
\mathrm{E}_{d}\left[\mathrm{E}_{\varphi}\left[\Delta \phi^{2}\right]\right] & =\frac{\mathrm{E}_{d}\left[d^{2}\right]}{2 R^{2}} \\
& =\frac{1}{N R^{2}} \\
& =\frac{\pi^{3}}{16 N \cdot \mathrm{SNR}} .
\end{aligned}
$$




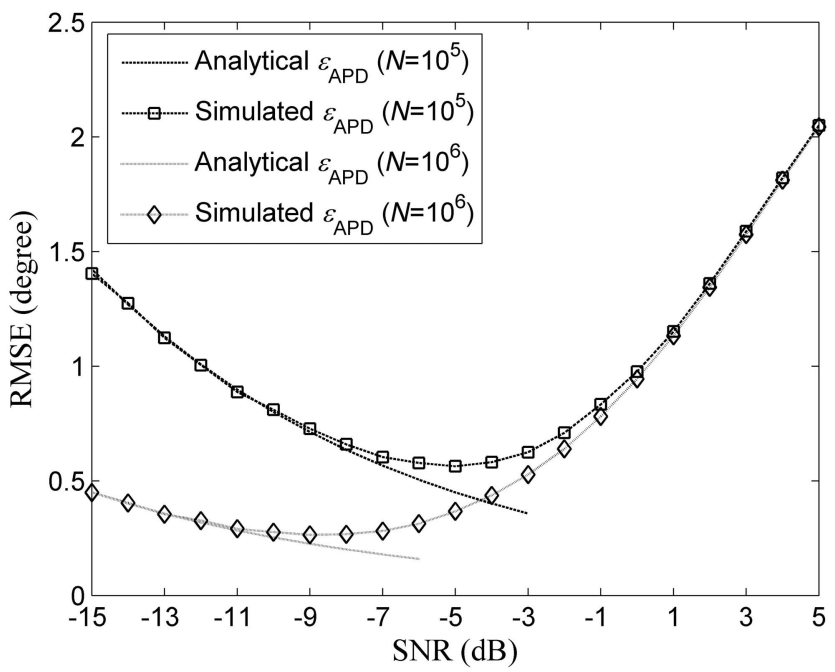

Fig. 5. Analytical and simulated results of APD regarding SNR.

In addition, the RMSE of APD is given by

$$
\begin{aligned}
\varepsilon_{\mathrm{APD}} & =\sqrt{\mathrm{E}_{d}\left[\mathrm{E}_{\phi}\left[\Delta \phi^{2}\right]\right]} \\
& =\frac{\pi^{3 / 2}}{4 \sqrt{N \cdot \mathrm{SNR}}} \quad \text { (radian) } \\
& =\frac{80}{\sqrt{N \cdot \mathrm{SNR}}} \quad \text { (degree) } .
\end{aligned}
$$

From (30), $\varepsilon_{\mathrm{APD}}$ is inversely proportional to the square root of the product of SNR and $N$. The analytical and simulated $\varepsilon_{\mathrm{APD}}$ are shown in Fig. 5. The simulated results are obtained by Monte Carlo simulation for 100 trials. From Fig. 5, the simulated results approach the analytical ones in low SNR. Note that the analytical $\varepsilon_{\mathrm{APD}}$ is provided in part since the result is valid only when low SNR is assumed. From Fig. 5, a minimal $\varepsilon_{\mathrm{APD}}$ exists at the specific SNR in the simulated cases. Below that $\mathrm{SNR}, \varepsilon_{\mathrm{APD}}$ increases with decreasing SNR, whereas $\varepsilon_{\mathrm{APD}}$ increases when the SNR is above that SNR. According to Fig. 2, since the relationship of the mean values of the I-Q outputs deviates from a circle with increasing SNR, the increase in $\varepsilon_{\mathrm{APD}}$ is due to the estimation bias of the APD, which is shown below.

\section{Moderate SNR}

Between the high SNR and the low SNR, the relationship between the mean I-Q outputs is complicated according to Fig. 2. The estimation bias of NB-DPD and APD is examined by means of the asymptotic performance. Suppose $N$ is sufficiently large. By LLN, the I-Q channel outputs of (15) approach their mean values, i.e., $I_{m} \rightarrow \mu_{I}$ and $Q_{m} \rightarrow$ $\mu_{Q}$. The asymptotic performance of NB-DPD and
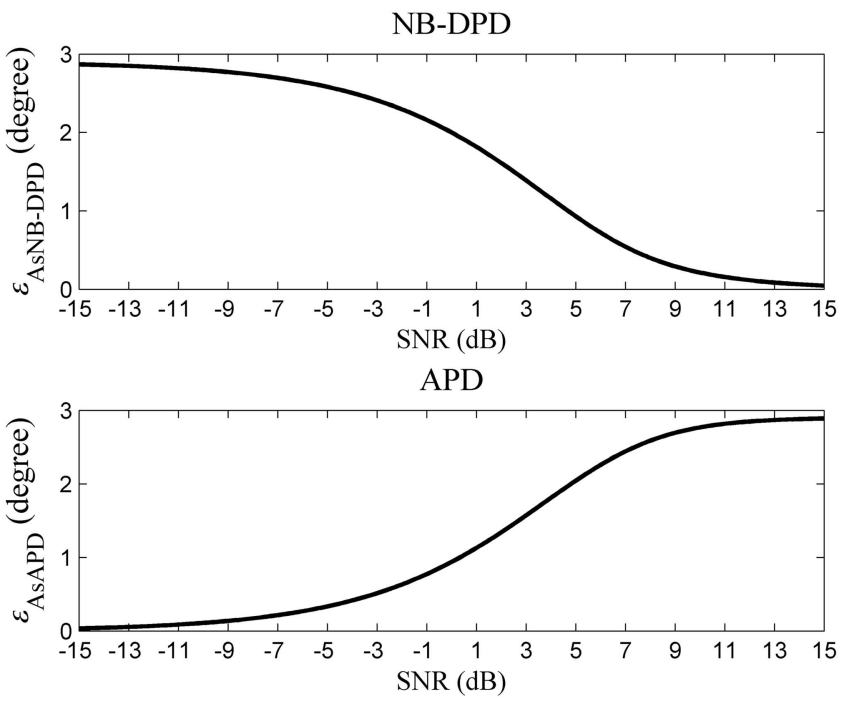

Fig. 6. Asymptotic performance of NB-DPD and APD regarding SNR.

APD are defined by

$$
\begin{aligned}
\hat{\phi}_{\mathrm{AsNB}-\mathrm{DPD}} & \triangleq \operatorname{sgn}\left(\mu_{Q}\right) \frac{\pi}{2}\left(1-\frac{\mu_{I}}{\left|\mu_{I}\right|+\left|\mu_{Q}\right|}\right) \\
\hat{\phi}_{\mathrm{AsAPD}} & \triangleq \operatorname{atan} 2\left(\frac{\mu_{Q}}{\mu_{I}}\right) .
\end{aligned}
$$

The corresponding squared phase errors are given by $e_{\mathrm{ND}}=\left(\hat{\phi}_{\mathrm{AsNB}-\mathrm{DPD}}-\phi\right)^{2}$ and $e_{\mathrm{A}}=\left(\hat{\phi}_{\mathrm{AsAPD}}-\phi\right)^{2}$, respectively. We further denote the asymptotic estimation error by

$$
\varepsilon_{\mathrm{AsNB}-\mathrm{DPD}}=\left(\bar{e}_{\mathrm{ND}}\right)^{1 / 2}
$$

and

$$
\varepsilon_{\text {AsAPD }}=\left(\bar{e}_{\mathrm{A}}\right)^{1 / 2} \text {. }
$$

Note that the averaged values of $e_{\mathrm{ND}}$ and $e_{\mathrm{A}}$, i.e., $\bar{e}_{\mathrm{ND}}$ and $\bar{e}_{\mathrm{A}}$, are used in (33) and (34), since they are functions of $\phi$. The $\varepsilon_{\mathrm{AsNB}-\mathrm{DPD}}$ and $\varepsilon_{\mathrm{AsAPD}}$ regarding SNR are shown in Fig. 6. From Fig. 6, $\varepsilon_{\text {AsNB-DPD }}$ and $\varepsilon_{\mathrm{AsAPD}}$ are small in high and low SNR, respectively. Specifically, $\varepsilon_{\text {AsNB-DPD }}<0.1 \mathrm{deg}$ if SNR $>12 \mathrm{~dB}$, and $\varepsilon_{\text {AsAPD }}<0.1$ deg when SNR $<-10 \mathrm{~dB}$. However, $\varepsilon_{\text {AsNB-DPD }}$ becomes significant when the SNR decreases whereas $\varepsilon_{\text {AsAPD }}$ becomes significant when the SNR increases. Here, the range of SNR in which both $\varepsilon_{\mathrm{AsNB}-\mathrm{DPD}}$ and $\varepsilon_{\mathrm{AsAPD}}$ are not negligible is considered "moderate" SNR. For example, SNR between $-10 \mathrm{~dB}$ and $12 \mathrm{~dB}$ in this case (phase accuracy requirement $<0.1 \mathrm{deg}$ ) is denoted as the moderate SNR. Obviously, for the moderate SNR, the accuracy of NB-DPD and APD are degraded and bounded by estimation bias, even though $N$ is sufficiently large. Note that even though the estimation error due to noise variance decreases with square root of SNR in APD as indicated in (30), the accuracy of APD is degraded because of estimation bias in moderate SNR. This result explains the degradation of $\varepsilon_{\mathrm{APD}}$ in moderate SNR as shown in Fig. 5. Above all, 
although NB-DPD and APD perform well in high and low SNR, respectively, their performance is degraded by the estimation bias in moderate SNR. Focusing on the moderate SNR, we develop an approach to reduce the estimation bias and thus improve the accuracy in the following section.

\section{SNR-AIDED PHASE DISCRIMINATOR}

\section{A. Proposed Approach}

Since the mean I-Q outputs vary with SNR as shown in Fig. 2, the SNR should be taken into consideration in achieving high-accuracy phase estimation. Therefore, the SNRaPD is developed to improve the accuracy by using the SNR information. Let $\left(\mu_{I}, \mu_{Q}\right)$ be the mean values of the I-Q output and $\left(I_{m}, Q_{m}\right)$ be the measured I-Q channel output. In SNRaPD, we define a measure by

$$
\begin{aligned}
L(\phi) & =\left(I_{m}-\mu_{I}\right)^{2}+\left(Q_{m}-\mu_{Q}\right)^{2} \\
& =\left(\lambda_{1}(\phi)\right)^{2}+\left(\lambda_{2}(\phi)\right)^{2}
\end{aligned}
$$

where $\lambda_{1}(\phi)=I_{m}-\mu_{I}, \lambda_{2}(\phi)=Q_{m}-\mu_{Q}$, and $\phi$ is the unknown phase to be estimated. In addition, let $\boldsymbol{\Lambda}(\phi)=\left[\lambda_{1}(\phi), \lambda_{2}(\phi)\right]^{\mathrm{T}}$, where "T" denotes transpose and $L(\phi)=\boldsymbol{\Lambda}(\phi)^{\mathrm{T}} \boldsymbol{\Lambda}(\phi)$. When the SNR information is given, $\gamma$ is known, and the relationship of $\left(\mu_{I}, \mu_{Q}\right)$ with respect to phase angle can be uniquely determined. When $\left(I_{m}, Q_{m}\right)$ are given, suppose the most likely phase is what minimizes $L(\phi)$ as follows:

$$
\hat{\phi}_{\mathrm{SNRaPD}}=\arg \min \{L(\phi)\} .
$$

This is the main idea of SNRaPD.

In optimization theory, the foregoing descriptions are known as the nonlinear least-square problem.

Newton's method can be used to search $\hat{\phi}=$ $\arg \min \{L(\phi)\}$ [18]. The vector of the first derivatives of $\lambda_{1}(\phi)$ and $\lambda_{2}(\phi)$ is given by

$$
\mathbf{J}(\phi)=\left[\begin{array}{ll}
\frac{d \lambda_{1}(\phi)}{d \phi} & \frac{d \lambda_{2}(\phi)}{d \phi}
\end{array}\right]^{\mathrm{T}} .
$$

In addition, let

$$
H(\phi)=\lambda_{1}(\phi) h_{1}(\phi)+\lambda_{2}(\phi) h_{2}(\phi)
$$

where

$$
\begin{aligned}
& h_{1}(\phi)=\frac{d^{2} \lambda_{1}(\phi)}{d \phi^{2}} \\
& h_{2}(\phi)=\frac{d^{2} \lambda_{2}(\phi)}{d \phi^{2}} .
\end{aligned}
$$

Then using Newton's method, $\phi$ is updated iteratively by

$$
\phi_{i}=\phi_{i-1}-\left(\mathbf{J}\left(\phi_{i-1}\right)^{\mathrm{T}} \mathbf{J}\left(\phi_{i-1}\right)+H\left(\phi_{i-1}\right)\right)^{-1} \mathbf{J}\left(\phi_{i-1}\right)^{\mathrm{T}} \boldsymbol{\Lambda}\left(\phi_{i-1}\right)
$$

where the $\phi_{i}$ denotes the phase obtained after the $i$ th iteration.
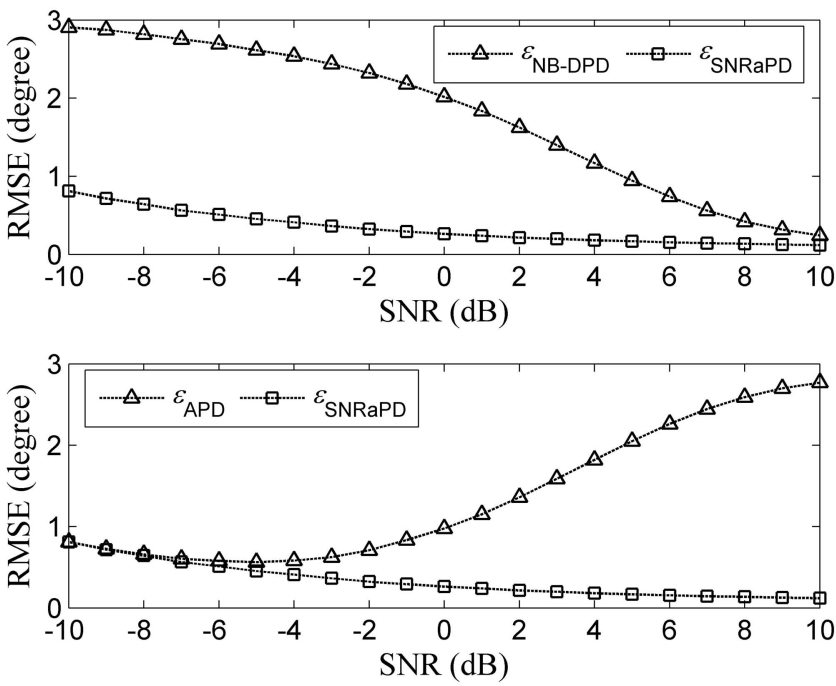

Fig. 7. Performance of SNRaPD regarding SNR.

In SNRaPD, depending on the available SNR information, the initial phase $\phi_{0}$ can be estimated by NB-DPD or APD, and accuracy can then be improved iteratively by (39). The iteration will stop when $\left|\phi_{i}-\phi_{i-1}\right|<\delta$, where $\delta$ is a small number. In the 1-bit SDR shown in Fig. 1, let the carrier frequency $f_{c}=15.421111 \mathrm{MHz}$, the sampling frequency $f_{s}=4.096 \mathrm{MHz}$, and $N=10^{5}$. In this case, according to (3), we have $p=4096000$ and $2 \pi / p$ will be significantly smaller than the estimation accuracy described later. The performance of SNRaPD is evaluated by Monte Carlo simulation. In the simulation, the stop criterion is $\delta=10^{-3} \mathrm{deg}$, and the estimated phase is given by $\hat{\phi}_{\mathrm{SNRaPD}}=\phi_{i}$. The RMSE defined by $\varepsilon_{\mathrm{SNRaPD}}=\sqrt{\mathrm{E}\left[\left(\hat{\phi}_{\mathrm{SNRaPD}}-\phi\right)^{2}\right]}$ is the average of 100 trials as shown in Fig. 7. From Fig. 7, the estimation errors by NB-DPD and APD are reduced with SNRaPD. For example, at $\mathrm{SNR}=0 \mathrm{~dB}$, the RMSE of NB-DPD is $2.017 \mathrm{deg}$ and that of SNRaPD is $0.2625 \mathrm{deg}$. A 7.7 times improvement is achieved. At SNR $=10 \mathrm{~dB}$, the RMSE of APD is $2.769 \mathrm{deg}$ and that of SNRaPD is $0.119 \mathrm{deg}$. A 23 times improvement is achieved. The proposed SNRaPD greatly improves the accuracy of phase estimation, especially in moderate SNR. Regarding the number of iterations of Newton's method in SNRaPD, normally less than four iterations are required from our simulation results. In addition, as mentioned in Section III, NB-DPD and APD perform well in high and low SNR, respectively. Hence, fewer iterations are required in SNRaPD when the initial phase is estimated by NB-DPD in high SNR and by APD in low SNR.

\section{B. Cramér-Rao Bound}

The CRB for 1-bit quantized complex-valued signals was derived in [11]. The performance of SNRaPD is compared with the CRB of the estimated 


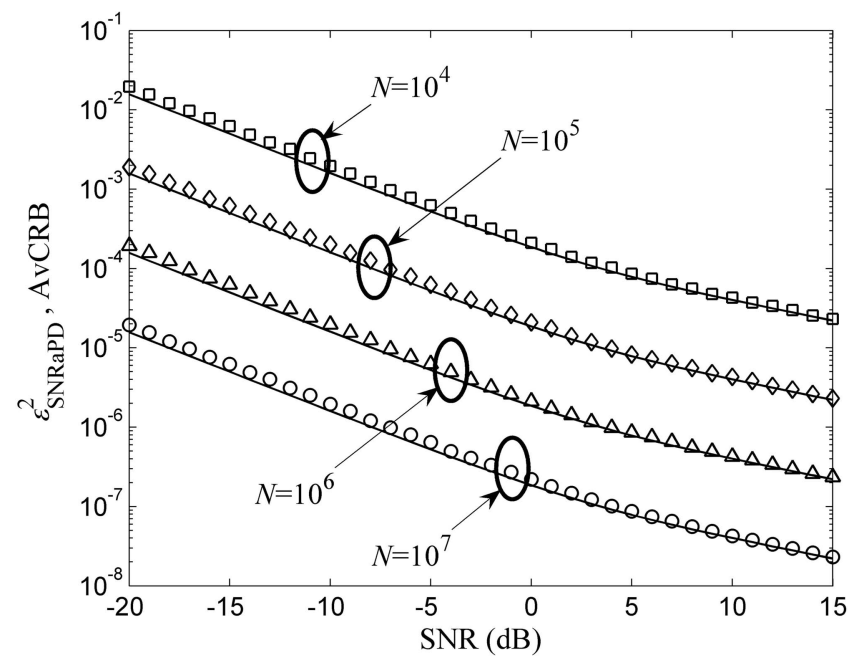

Fig. 8. MSE of SNRaPD (markers) and AvCRB (solid lines).

phase. For simplicity, we only consider the real-value case. The probability mass function of $r[k]$ in (2) is given by

$$
\begin{aligned}
f_{r}(q ; \phi) & =\operatorname{Pr}(q \cdot r[k]>0 ; \phi) \\
& =\frac{1}{\sqrt{2 \pi} \sigma} \int_{0}^{\infty} \exp \left(-\frac{\left(r-q \cdot \sin \left(\Phi_{k}+\phi\right)\right)^{2}}{2 \sigma^{2}}\right) d r .
\end{aligned}
$$

The Fisher information is then obtained by

$$
\begin{aligned}
\mathrm{I}(\phi) & =\sum_{k=0}^{N-1} \sum_{q= \pm 1} \frac{1}{f_{r}(q ; \phi)} \frac{\partial f_{r}}{\partial \phi}(q ; \phi) \frac{\partial f_{r}}{\partial \phi}(q ; \phi)^{T} \\
& =\frac{1}{2 \pi \sigma^{2}} \sum_{k=0}^{N-1} \psi\left(\Phi_{k}+\phi ; 1 / \sigma\right)
\end{aligned}
$$

where

$$
\begin{aligned}
\psi\left(\Phi_{k}+\phi ; 1 / \sigma\right) & =\frac{4 \cos ^{2}\left(\Phi_{k}+\phi\right) \cdot \exp \left(-\frac{1}{\sigma^{2}} \sin ^{2}\left(\Phi_{k}+\phi\right)\right)}{1-\operatorname{erf}\left((1 / \sqrt{2} \sigma) \cdot \sin \left(\Phi_{k}+\phi\right)\right)^{2}} \\
\operatorname{erf}(x) & =\frac{2}{\sqrt{\pi}} \int_{0}^{x} \exp \left(-t^{2}\right) d t .
\end{aligned}
$$

To investigate the achievable performance of SNRaPD, we assume that the frequency in (41) is zero, i.e., $\Phi_{k}=0$, and the phase is a uniform random variable. We define the average Fisher information by averaging (41) over phases, which is denoted by

$$
\begin{aligned}
\overline{\mathrm{I}} & =\frac{1}{2 \pi} \int_{0}^{2 \pi} \mathrm{I}(\phi) d \phi \\
& =\frac{N}{2 \pi \sigma^{2}} \bar{\psi}(1 / \sigma)
\end{aligned}
$$

where $\bar{\psi}(1 / \sigma)=(1 / 2 \pi) \int_{0}^{2 \pi} \psi(\phi ; 1 / \sigma) d \phi$.

Taking the inverse of the average Fisher information, we have the average CRB given by

$$
\mathrm{AvCRB}=\frac{\pi}{N \cdot \operatorname{SNR} \cdot \bar{\psi}(1 / \sigma)}
$$

where SNR is defined by (9).
The MSE of SNRaPD defined by $\varepsilon_{\text {SNRaPD }}^{2}=$ $\mathrm{E}\left[\left(\hat{\phi}_{\mathrm{SNRaPD}}-\phi\right)^{2}\right]$ is compared with the AvCRB in Fig. 8. The AvCRB is plotted with solid lines, and the $\varepsilon_{\mathrm{SNRaPD}}^{2}$ is denoted by markers. The performance of SNRaPD is obtained from Monte Carlo simulation using 100 trials. In Fig. $8, \varepsilon_{\text {SNRaPD }}^{2}$ is close to AvCRB and their difference approaches zero with increasing SNR. Thus the accuracy of SNRaPD is certified. Note that the difference between AvCRB and $\varepsilon_{\mathrm{SNRaPD}}^{2}$ is similar for different values of $N$.

\section{Stop Criterion}

As mentioned at the end of Section IVA, although estimation bias is reduced by SNRaPD, and $\hat{\phi}_{\mathrm{SNRaPD}}=$ $\arg \min \{L(\phi)\}$ is achieved by Newton's method, noise variance still exists in $\hat{\phi}_{\mathrm{SNRaPD}}$. As a result, the strict criterion $\left|\phi_{i}-\phi_{i-1}\right|<10^{-3}(\mathrm{deg})$ may result in a need for extra iterations, but the performance cannot be improved. From (35), the first derivative of $L(\phi)$ is given by

$$
\begin{aligned}
\frac{d L(\phi)}{d \phi} & =2\left[\lambda_{1}(\phi) \frac{d \lambda_{1}(\phi)}{d \phi}+\lambda_{2}(\phi) \frac{d \lambda_{2}(\phi)}{d \phi}\right] \\
& =2 \mathbf{J}^{\mathrm{T}}(\phi) \Lambda(\phi) .
\end{aligned}
$$

Note that the original criterion $\left|\phi_{i}-\phi_{i-1}\right|<10^{-3}$ is consistent with $d L\left(\hat{\phi}_{\mathrm{SNRaPD}}\right) / d \phi=0$, since $\hat{\phi}_{\mathrm{SNRaPD}}=$ $\arg \min \{L(\phi)\}$. As we consider the noise variance with (44), a new stop criterion is defined for SNRaPD as follows. Let $\phi=\phi_{c}+\Delta \phi_{c}$ in (44), where $\phi_{c}$ is the true phase, and $\Delta \phi_{c}$ is the phase error due to noise variance. Since the AvCRB of (43) is the theoretical bound of variance, let $\Delta \phi_{c}=\sqrt{\mathrm{AvCRB}}$. The new stop criterion is defined by

$$
\left|2 \mathbf{J}\left(\phi_{i}\right)^{\mathrm{T}} \boldsymbol{\Lambda}\left(\phi_{i}\right)\right|<\left|2 \mathbf{J}\left(\phi_{c}+\Delta \phi_{c}\right)^{\mathrm{T}} \boldsymbol{\Lambda}\left(\phi_{c}+\Delta \phi_{c}\right)\right| .
$$

Note that the absolute value is used since the same result but with opposite polarity is obtained by $\phi=\phi_{c}-\Delta \phi_{c}$. In addition, the criterion $\left|2 \mathbf{J}\left(\phi_{c}+\Delta \phi_{c}\right)^{\mathrm{T}} \boldsymbol{\Lambda}\left(\phi_{c}+\Delta \phi_{c}\right)\right|$ is a function of $\phi_{c}$, and the minimal value occurs when $\phi_{c}=k \pi / 2$. Hence, the criterion is further defined by $\mid 2 \mathbf{J}\left(\phi_{c}+\Delta \phi_{c}\right)^{\mathrm{T}} \boldsymbol{\Lambda}$ $\left.\cdot\left(\phi_{c}+\Delta \phi_{c}\right)\right|_{\phi_{c}=k \pi / 2}$ to guarantee that the criterion is valid for all phases.

Example. Determination of the Stop Criterion: Let SNR $=6 \mathrm{~dB}, N=10^{5}$, and $\phi_{c}=0^{\circ}$. According to (43), $\Delta \phi_{c}=\sqrt{\mathrm{AvCRB}}=0.12215^{\circ}$. Applying $\phi=\phi_{c}+\Delta \phi_{c}=0.12215^{\circ}$ to (44), we have $\left|2 \mathbf{J}\left(0.12215^{\circ}\right)^{\mathrm{T}} \boldsymbol{\Lambda}\left(0.12215^{\circ}\right)\right|=0.001712$. Hence, the iteration will stop when $\left|2 \mathbf{J}\left(\phi_{i}\right)^{\mathrm{T}} \boldsymbol{\Lambda}\left(\phi_{i}\right)\right|<0.001712$.

A similar RMSE is achieved with the new stop criterion according to the simulation results, which verifies our supposition. Moreover, the number of iterations is reduced with the new criterion. Recall that the original criterion is consistent with $d L\left(\hat{\phi}_{\text {SNRaPD }}\right) / d \phi=0$. Since Newton's method 

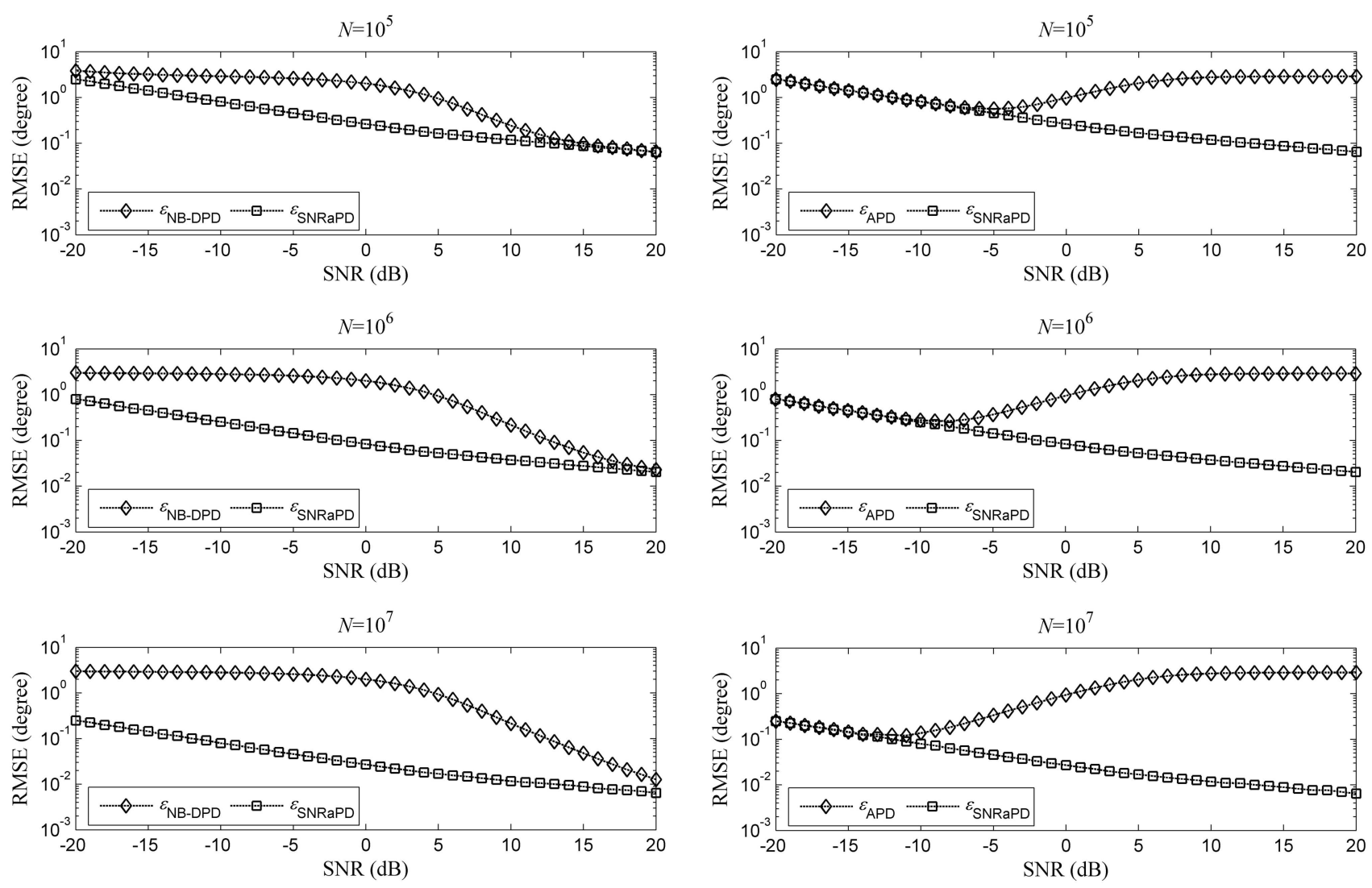

(a)

(b)

Fig. 9. Comparison of RMSE of SNRaPD and that of NB-DPD and APD.

will reach the criterion of $d L(\phi) / d \phi=2 \mid \mathbf{J}^{\mathrm{T}}\left(\phi_{c}+\right.$ $\left.\Delta \phi_{c}\right) \boldsymbol{\Lambda}\left(\phi_{c}+\Delta \phi_{c}\right) \mid$ before that of $d L\left(\hat{\phi}_{\mathrm{SNRaPD}}\right) / d \phi=0$, the number of iterations is reduced accordingly.

Finally, most computational loads of SNRaPD fall in computing $\lambda_{1}(\phi)$ and $\lambda_{2}(\phi)$ as well as their derivatives, which involve $\mu_{I}$ and $\mu_{Q}$ of (11) and (13), respectively. In practice, we use $M$ terms to approximate $\mu_{I}$ and $\mu_{Q}$ rather than infinite sums. Moreover, we can calculate and store coefficients in a table in order to further mitigate the computational burdens. For applications with SNR less than $0 \mathrm{~dB}$, $M=8$ is sufficient to well approximate $\mu_{I}$ and $\mu_{Q}$ with RMSE smaller than $10^{-6}$. In addition, less than four iterations are normally required from our simulation results. Therefore, the computational burden of SNRaPD is feasible for today's fast processors.

\section{Range of Application}

The range of application of SNRaPD is studied by comparing $\varepsilon_{\mathrm{SNRaPD}}$ with $\varepsilon_{\mathrm{NB}-\mathrm{DPD}}$ and $\varepsilon_{\mathrm{APD}}$. The simulated results regarding SNR and $N$ are illustrated in Fig. 9. From Fig. 9(a), compared with NB-DPD, the improvement in the accuracy of SNRaPD is significant in moderate SNR. Note that the range of the moderate SNR may vary according to $N$ and the required phase accuracy in different applications. Moreover, in $N=10^{5}$ and $N=10^{6}$ cases, the improvement is negligible when the SNR is above
$12 \mathrm{~dB}$ and $17 \mathrm{~dB}$, respectively. As $N$ increases to $10^{7}, \mathrm{SNRaPD}$ can consistently improve the accuracy to some degree for SNR below $20 \mathrm{~dB}$. Similarly, in comparing SNRaPD with APD, the accuracy is greatly improved in moderate to high SNR as shown in Fig. 9(b). The improvement is negligible when the SNR is below $-4 \mathrm{~dB},-8 \mathrm{~dB}$, and $-12 \mathrm{~dB}$, respectively.

The above discussion illustrates the superiority of the proposed SNRaPD in moderate SNR. As the ambient SNR is increased to the moderate range, the SNRaPD can potentially be used and provide improved accuracy in spaceborne measurement techniques, such as TEC measurements on a beacon receiver or the POD on a GNSS receiver [19-23]. For example, when $N=10^{6}$ and SNR $=0 \mathrm{~dB}$ on the $400 \mathrm{MHz}$ beacon signal, the phase error is $0.08 \mathrm{deg}$ and $0.94 \mathrm{deg}$ for SNRaPD and APD, respectively. The estimation error of SNRaPD is approximately an order better than that of APD. If more samples are allowed, the superiority of SNRaPD over APD remains significant even when the SNR is lower than $0 \mathrm{~dB}$ as shown in Fig. 9(b).

\section{CONCLUSIONS}

In this paper, the high-accuracy phase estimation for 1-bit SDR is investigated. Specifically, the received signal and local reference signal are both 
1-bit quantized for efficient bitwise processing in 1-bit SDR. We propose SNRaPD using the SNR information to reduce the estimation bias and achieve the high-accuracy phase estimation. The mean values and variances of the I-Q channel outputs in 1-bit SDR are given by (11), (13), and (16), and the SNR-dependent relationship of the mean I-Q channel outputs is explicitly shown in Fig. 2. For high-accuracy phase estimation, NB-DPD can be used in high SNR as shown in Fig. 3. For the low SNR, APD is selected according to (20), and its performance is illustrated in Fig. 5. However, according to the asymptotic performance of NB-DPD and APD as shown in Fig. 6, the estimation bias becomes significant and can have a negative effect on the accuracy in moderate SNR. Focusing on this SNR range, we proposed SNRaPD using Newton's method to reduce bias and improve the resulting accuracy as shown in Fig. 7. The accuracy of SNRaPD is certified by comparing the MSE with the AvCRB in Fig. 8. In order to save the computation, an adequate stop criterion for SNRaPD with concerning noise variance is also defined by (45).

In addition, further studies on the accuracy of SNRaPD may be needed when the SNR estimation error and the frequency offset appear in realistic applications. Generally, the error in SNR estimation is small in high SNR while the SNRaPD is less sensitive to the SNR error in low to moderate SNR, which has been shown in Fig. 2. On the other hand, the frequency offset not only causes variations in phase estimation but also affects the achievable accuracy, i.e., $2 \pi / p$ as mentioned in Section II.

Finally, the range of the application of SNRaPD is investigated by comparing the associated RMSE with that of NB-DPD and APD as shown in Fig. 9. Potential applications of SNRaPD and the corresponding performance on $\mathrm{SNR}=0 \mathrm{~dB}$ are also mentioned. It is worthwhile to mention that the range of application of SNRaPD (moderate SNR) expands with increasing $N$. Note that because of the simplicity of our signal model, e.g., ignoring the frequency variation, the limitation of integration time, and the mismatch of PN codes, the actual improvement in the beacon signal measurement or the GNSS signal tracking may need further investigation. Furthermore, since the proposed SNRaPD requires the knowledge of SNR which is not available in many applications, the joint phase and SNR estimation is needed. This will be our next step.

\section{APPENDIX I. DERIVATION OF MEAN AND VARIANCE OF I-Q CHANNEL OUTPUTS}

According to (4), for $\Phi_{k} \in[0, \pi)$, we have $\sin \Phi_{k} \geq$ 0 . In inphase (I) channel, the conditional probabilities are denoted as

$$
\begin{aligned}
\operatorname{Pr}\left(a_{k}=1 \mid \Phi_{k}\right) & =\operatorname{Pr}\left(\sin \left(\Phi_{k}+\phi\right)+\nu_{k} \geq 0 \mid \Phi_{k}\right) \\
& =1-\mathrm{P}_{k} \\
\operatorname{Pr}\left(a_{k}=-1 \mid \Phi_{k}\right) & =\operatorname{Pr}\left(\sin \left(\Phi_{k}+\phi\right)+\nu_{k}<0 \mid \Phi_{k}\right) \\
& =\mathrm{P}_{k} .
\end{aligned}
$$

Thus the mean and variance of $a_{k}$ are given by

$$
\begin{aligned}
\mu_{a_{k}} & =1 \cdot \operatorname{Pr}\left(a_{k}=1 \mid \Phi_{k}\right)+(-1) \cdot \operatorname{Pr}\left(a_{k}=-1 \mid \Phi_{k}\right) \\
& =1-2 \mathrm{P}_{k} \\
\sigma_{a_{k}}^{2} & =\mathrm{E}\left[a_{k}^{2}\right]-\mu_{a_{k}}^{2} \\
& =1-\left(1-2 \mathrm{P}_{k}\right)^{2} \\
& =4\left(\mathrm{P}_{k}-\mathrm{P}_{k}^{2}\right)
\end{aligned}
$$

Similarly, for $\Phi_{k} \in[\pi, 2 \pi)$, we have $\sin \Phi_{k} \leq 0$. The conditional probabilities are denoted as

$$
\begin{aligned}
\operatorname{Pr}\left(a_{k}=1 \mid \Phi_{k}\right) & =\operatorname{Pr}\left(\sin \left(\Phi_{k}+\phi\right)+\nu_{k}<0 \mid \Phi_{k}\right) \\
& =\mathrm{P}_{k} \\
\operatorname{Pr}\left(a_{k}=-1 \mid \Phi_{k}\right) & =\operatorname{Pr}\left(\sin \left(\Phi_{k}+\phi\right)+\nu_{k} \geq 0 \mid \Phi_{k}\right) \\
& =1-\mathrm{P}_{k} .
\end{aligned}
$$

The associated mean and variance are given by

$$
\begin{aligned}
\mu_{a_{k}} & =2 \mathrm{P}_{k}-1 \\
\sigma_{a_{k}}^{2} & =4\left(\mathrm{P}_{k}-\mathrm{P}_{k}^{2}\right) .
\end{aligned}
$$

Assume the noise component in each sample is independent. Since $\Phi_{k}$ s are uniformly distributed over $[0,2 \pi)$, we have

$$
\begin{aligned}
\mu_{I_{p}} & =\frac{1}{p}\left[\sum_{\Phi_{k} \in[0, \pi)}\left(1-2 \mathrm{P}_{k}\right)+\sum_{\Phi_{k} \in[\pi, 2 \pi)}\left(2 \mathrm{P}_{k}-1\right)\right] \\
\sigma_{I}^{2} & =\frac{4}{p^{2}} \sum_{k=0}^{p-1} \mathrm{P}_{k}-\mathrm{P}_{k}^{2} .
\end{aligned}
$$

Similarly, by the same calculation, the mean and variance for the quadrature $(\mathrm{Q})$ channel are obtained by

$$
\begin{aligned}
\mu_{Q_{p}} & =\frac{1}{p}\left[\sum_{\Phi_{k} \in[0, \pi / 2) \cup[3 \pi / 2,2 \pi)}\left(1-2 \mathrm{P}_{k}\right)+\sum_{\Phi_{k} \in[\pi / 2,3 \pi / 2)}\left(2 \mathrm{P}_{k}-1\right)\right] \\
\sigma_{Q}^{2} & =\frac{4}{p^{2}} \sum_{k=0}^{p-1} \mathrm{P}_{k}-\mathrm{P}_{k}^{2} .
\end{aligned}
$$


APPENDIX II. POWER SERIES REPRESENTATION OF MEAN AND VARIANCE OF I-Q CHANNEL OUTPUTS

The power series representation of the Q-function is given by [17]

$$
\mathrm{Q}(x)=\frac{1}{2}-\frac{1}{\sqrt{2 \pi}} \sum_{m=0}^{\infty} \frac{(-1)^{m} x^{2 m+1}}{m ! 2^{m}(2 m+1)} .
$$

\section{REFERENCES}

[1] Chang, C-F., Yang, R-M., and Kao, M-S.

Implementation of an innovative phase discriminator for improved tracking performance in one-bit software GPS receiver.

Proceedings of the National Technical Meeting of the ION, San Diego, CA, Jan. 2008.

Hence, (10) is rewritten by

$$
\begin{aligned}
\mu_{I_{p}} & =\frac{1}{p}\left\{\sum_{\Phi_{k} \in[\pi, 2 \pi)}\left[1-\frac{2}{\sqrt{2 \pi}} \sum_{m=0}^{\infty} \frac{(-1)^{m}\left(\gamma \sin \left(\Phi_{k}+\phi\right)\right)^{2 m+1}}{m ! 2^{m}(2 m+1)}\right]-\sum_{\Phi_{k} \in[0, \pi)}\left[1-\frac{2}{\sqrt{2 \pi}} \sum_{m=0}^{\infty} \frac{(-1)^{m}\left(\gamma \sin \left(\Phi_{k}+\phi\right)\right)^{2 m+1}}{m ! 2^{m}(2 m+1)}\right]\right\} \\
& =\sqrt{\frac{2}{\pi}} \sum_{m=0}^{\infty} \frac{(-1)^{m} \gamma^{2 m+1}}{m ! 2^{m}(2 m+1)}\left\{\frac{1}{p}\left[\sum_{\Phi_{k} \in[0, \pi)} \sin \left(\Phi_{k}+\phi\right)^{2 m+1}-\sum_{\Phi_{k} \in[\pi, 2 \pi)} \sin \left(\Phi_{k}+\phi\right)^{2 m+1}\right]\right\} .
\end{aligned}
$$

Suppose we choose $f_{s}$ such that $p$ is sufficiently large

in (3), (59) is approximated by

$$
\mu_{I_{p}} \cong \sqrt{\frac{2}{\pi}} \sum_{m=0}^{\infty} \frac{(-1)^{m} \gamma^{2 m+1}}{m ! 2^{m}(2 m+1)}\left\{\frac{1}{2 \pi}\left[\int_{0}^{\pi} \sin ^{2 m+1}(\Phi+\phi) d \Phi-\int_{\pi}^{2 \pi} \sin ^{2 m+1}(\Phi+\phi) d \Phi\right]\right\}
$$

where $d \Phi=2 \pi / p \rightarrow 0$. By the power series

representation of the integrand involving the odd power of $\sin (x)$ [17, sec. 9.2.1], (60) can be further written as

$$
\mu_{I_{p}}=\frac{4}{\sqrt{2} \pi^{3 / 2}} \sum_{m=0}^{\infty} \frac{\gamma^{2 m+1}}{m ! 2^{3 m}(2 m+1)}\left[\sum_{l=0}^{m}(-1)^{l}\left(\begin{array}{c}
2 m+1 \\
l
\end{array}\right) \frac{\cos (2 m+1-2 l) \phi}{2 m+1-2 l}\right] .
$$

The derivation of the mean value of the Q-channel output is omitted because of similarity. In addition, using (58), the variances of the I-Q channel outputs of (7) are derived by

$$
\begin{aligned}
\sigma_{I_{p}}^{2} & =\sigma_{Q_{p}}^{2} \\
& =\frac{4}{p^{2}} \sum_{k=0}^{p-1}\left\{\left[\frac{1}{2}-\frac{1}{\sqrt{2 \pi}} \sum_{m=0}^{\infty} \frac{(-1)^{m}\left(\gamma \sin \left(\Phi_{k}+\phi\right)\right)^{2 m+1}}{m ! 2^{m}(2 m+1)}\right]-\left[\frac{1}{2}-\frac{1}{\sqrt{2 \pi}} \sum_{m=0}^{\infty} \frac{(-1)^{m}\left(\gamma \sin \left(\Phi_{k}+\phi\right)\right)^{2 m+1}}{m ! 2^{m}(2 m+1)}\right]^{2}\right\} \\
& =\frac{1}{p}-\frac{2}{p^{2} \pi} \sum_{k=0}^{p-1}\left[\sum_{m=0}^{\infty} \frac{\left(\gamma \sin \left(\Phi_{k}+\phi\right)\right)^{4 m+2}}{\left(m ! 2^{m}(2 m+1)\right)^{2}}+2 \sum_{x=0}^{\infty} \sum_{y=x+1}^{\infty} \frac{(-1)^{x+y}\left(\gamma \sin \left(\Phi_{k}+\phi\right)\right)^{2(x+y)+2}}{x ! y ! 2^{x+y}(2 x+1)(2 y+1)}\right]^{2} \\
& \cong \frac{1}{p}-\frac{2}{p \pi} \cdot \frac{1}{2 \pi} \int_{0}^{2 \pi}\left[\sum_{m=0}^{\infty} \frac{(\gamma \sin (\Phi+\phi))^{4 m+2}}{\left(m ! 2^{m}(2 m+1)\right)^{2}}+2 \sum_{x=0}^{\infty} \sum_{y=x+1}^{\infty} \frac{(-1)^{x+y}(\gamma \sin (\Phi+\phi))^{2(x+y)+2}}{\left(x ! y ! 2^{x+y}(2 x+1)(2 y+1)\right.}\right] d \Phi .
\end{aligned}
$$

By the power series representation of the integrand involving the even power of $\sin (x)$ [17, sec. 9.2.1], (62) can be further written as

$$
\begin{aligned}
\sigma_{I_{p}}^{2} & =\sigma_{Q_{p}}^{2} \\
& =\frac{1}{p}-\frac{2}{p \pi}\left[\sum_{m=0}^{\infty}\left(\begin{array}{c}
4 m+2 \\
2 m+1
\end{array}\right) \frac{\gamma^{4 m+2}}{\left(m ! 2^{3 m+1}(2 m+1)\right)^{2}}+2 \sum_{x=0}^{\infty} \sum_{y=x+1}^{\infty}\left(\begin{array}{c}
2 x+2 y+2 \\
x+y+1
\end{array}\right) \frac{(-1)^{x+y} \gamma^{2(x+y)+2}}{x ! y ! 2^{3(x+y)+2}(2 x+1)(2 y+1)}\right] .
\end{aligned}
$$


[2] Kay, S.

Fundamentals of Statistical Signal Processing: Estimation Theory, vol. 1 .

Upper Saddle River, NJ: Prentice-Hall, 1993.

[3] Papadopoulos, H. C., Wornell, G. W., and Oppenheim, A. V. Low-complexity digital encoding strategies for wireless sensor networks.

Proceedings of the IEEE International Conference on Acoustic, Speech and Signal Processing, Seattle, WA, May 1998.

[4] Wu, P. H.

The optimal BPSK demodulator with a 1-bit A/D front-end.

Proceedings of the IEEE Military Communications Conference, Boston, MA, Oct. 1998.

[5] Brown, A. and Wolt, B.

Digital L-band receiver architecture with direct RF sampling.

Proceedings of the IEEE Position Location and Navigation Symposium, Las Vegas, NV, Apr. 1994, 209-216.

[6] Walden, R. H.

Analog-to-digital converter survey and analysis. IEEE Journal on Selected Areas in Communications, 17, 4 (Apr. 1999), 539-550.

[7] Ledvina, B. M., et al.

A real-time GPS civilian L1/L2 software receiver.

Proceedings of the International Technical Meeting of the Satellite Division of the ION, Long Beach, CA, Sept. 2004.

[8] Rife, D. and Boorstyn, R.

Single tone parameter estimation from discrete-time observations.

IEEE Transactions on Information Theory, IT-20 (Oct. 1974), 591-598.

[9] Quinn, B. G.

Estimation of frequency, amplitude, and phase from the DFT of a time series.

IEEE Transactions on Signal Processing, 45, 3 (Mar. 1997), 814-817.

[10] Fu, H. and Kam, P. Y.

MAP/ML estimation of the frequency and phase of a single sinusoid in noise.

IEEE Transactions on Signal Processing, 55, 3 (Mar. 2007), 834-845.

[11] Host-Madsen, A. and Handel, P.

Effects of sampling and quantization on single-tone frequency estimation.

IEEE Transactions on Signal Processing, 48, 3 (Mar. 2000), 650-662.

[12] Dabeer, O. and Karnik, A.

Signal parameter estimation using 1-bit dithered quantization.

IEEE Transactions on Information Theory, 52, 12 (Dec. 2006), 5389-5405.
[13] Dabeer, O. and Masry, E.

Multivariate signal parameter estimation under dependent noise from 1-bit dithered quantized data.

IEEE Transactions on Information Theory, 54, 4 (Apr. 2008), 1637-1654.

[14] Pouzet, A. H.

Characteristics of phase detectors in presence of noise. Proceedings of the International Telemeter Conference, Los Angeles, CA, 1972.

[15] Gardner, F. M.

Phaselock Techniques.

Hoboken, NJ: Wiley-Interscience, 2005.

[16] Chang, C-F. and Kao, M-S.

High-accuracy carrier phase discriminator for one-bit quantized software-defined receivers. IEEE Signal Processing Letters, 15 (2008), 397-400.

[17] Jeffrey, A.

Handbook of Mathematical Formulas and Integrals. Amsterdam: Elsevier, 2004.

[18] Chong, E. K. P. and Zak, S. H.

An Introduction to Optimization

Hoboken, NJ: Wiley-Interscience, 2008.

[19] Bernhardt, P. A., et al.

Ionospheric applications of the scintillation and tomography receiver in space (CITRIS) mission when used with the DORIS radio beacon network.

Journal of Geodesy, 80 (2006), 473-485.

[20] Kikkert, C. J. and Kenny, O. P.

A digital signal processing based Ka band satellite beacon receiver.

Proceedings of the IEEE International Conference on Electronics, Circuits, and Systems, St. Julien's, Malta, 2008, 598-601.

[21] Parkinson, B. W. and Spilker, J. J.

Global Positioning System: Theory and Applications, vol. II.

Washington, DC: AIAA, 1996, ch. 21.

[22] Montenbruck, O., Garcia-Fernandez, M., and Williams, J. Performance comparison of semicodeless GPS receivers for LEO satellites.

GPS Solutions, 10, 4 (2006), 249-261.

[23] Huwang, C-W., et al.

Quality assessment of FORMOSAT-3/COSMIC and GRACE GPS observables: Analysis of multipath, ionospheric delay and phase residual in orbit determination.

GPS Solutions, 14, 1 (Jan. 2010), 121-131. 

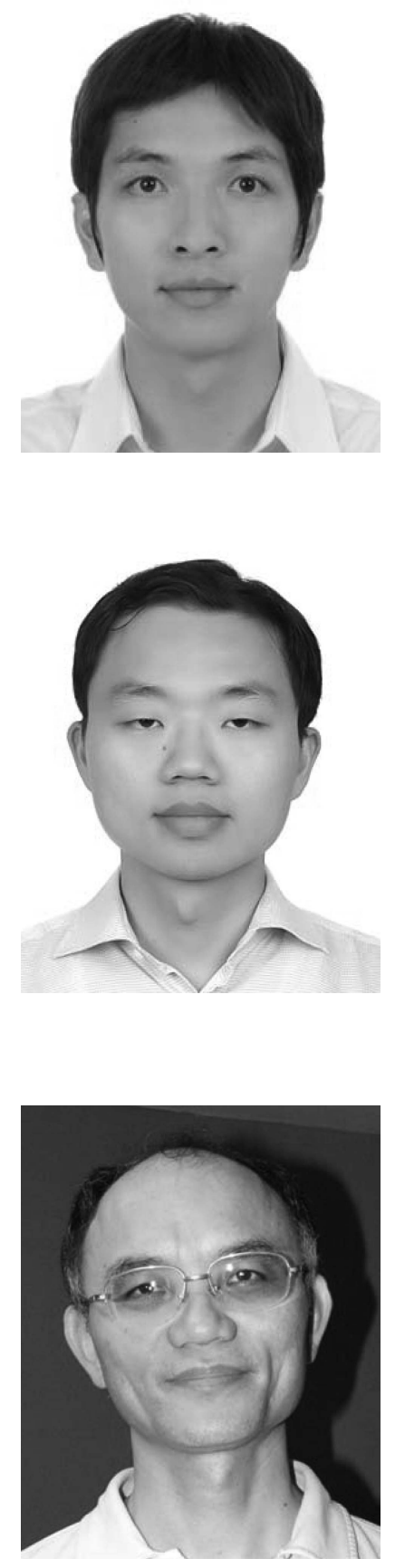

Wan-Hsin Hsieh received the B.S. and the M.S. degrees from National Chiao-Tung University, Taiwan, where he is currently a Ph.D. student in the Department of Electrical Engineering.

$\mathrm{He}$ is interested in digital signal processing and parameter estimation.

Chieh-Fu Chang received his $\mathrm{Ph} . \mathrm{D}$. in electrical and computer engineering, from Purdue University, West Lafayette, IN.

$\mathrm{He}$ is currently an assistant researcher at the Electrical Engineering Division of National Space Organization (NSPO), Taiwan. His current research interests include software-defined receivers, digital signal processing, communications, radar and optical remote sensing instruments.

Ming-Seng Kao (S'89-M'90) was born in Taipei, Taiwan, R.O.C., in 1959. He received the B.S.E.E. degree from National Taiwan University, in 1982, the M.S. degree in optoelectronics from National Chiao-Tung University, in 1986, and the Ph.D. degree in electrical engineering from National Taiwan University, in 1990.

From 1986 to 1987 he was an assistant researcher at the Telecommunications Laboratories, Chung-Li, Taiwan. In 1990, he joined the faculty of National Chiao Tung University, Hsinchu, Taiwan, where he is now a professor in the Communication Engineering Department. Between 1993-1994, he was a visiting professor at the Swiss Federal Institute of Technology (ETH), Zurich, Switzerland, where he worked in the area of optical communications. In 2003 he was a visiting professor at the Nanyang Technological University, Singapore, where he investigated optical devices and networking. He is currently interested in wireless communications and digital receivers. 